\title{
Probing new physics with high-multiplicity events: Ultrahigh-energy neutrinos at air-shower detector arrays
}

\author{
Yongsoo Jho* and Seong Chan Park ${ }^{\dagger}$ \\ Department of Physics and IPAP, Yonsei University, Seoul 03722, Republic of Korea
}

(Received 1 November 2020; accepted 22 June 2021; published 14 July 2021)

\begin{abstract}
Semiclassical processes such as production and decay of electroweak sphaleron in the Standard Model and also microscopic black hole in low scale gravity scenario typically involve large number of particles in final states. These large multiplicities can be distinctively seen in collisions of ultra-high-energy (UHE) neutrinos with $E_{\nu} \gtrsim 10^{9} \mathrm{GeV}$ and nucleons in the atmosphere of the Earth. Focusing on air-shower detector array experiments including Telescope Array Experiment (TA), Pierre-Auger Observatory (Auger), we propose strategic ways to discover and analyze such events.
\end{abstract}

DOI: 10.1103/PhysRevD.104.015018

\section{INTRODUCTION}

Recent observation of ultra-high-energy cosmic rays (UHECRs) extended the domain of the high-energy frontier covering beyond the reach of collider experiments. Many $\mathcal{O}(10) \mathrm{EeV}$ cosmic ray events and a few $\mathcal{O}(1) \mathrm{PeV}$ neutrino events have been already observed by ground airshower detector arrays [1,2] and other large detectors [3]. There are widely accepted and also more speculative potential origins of such UHECRs: young magnetized neutron star [4], active galactic nuclei (AGN) [5], gamma-ray burst (GRB) [6,7], BL Lac type objects [8], dark matter, and topological defects [9], but none of them have been confirmed yet. Furthermore, the fluxes, composition, and the production mechanisms of UHECRs sensitively depend on the origin. Thus, they still need to be clarified [10-15]. From the observation of UHECRs up to $E_{\mathrm{CR}} \sim 10^{21} \mathrm{eV}$, we also naturally expect the guaranteed existence of ultra-high-energy (UHE) neutrinos, produced by the Greisen-Zatsepin-Kuzmin (GZK) mechanism between UHECR nuclei and the cosmic-microwave-background $(\mathrm{CMB})$ photon during extragalactic propagation.

Even though uncertainties are still persist, our attention focuses on scattering process of UHECR and UHE neutrino with known targets, such as the nucleons in the atmosphere. We anticipate that such a "fixed-target" experiment can provide a testing ground for particle physics beyond $\mathrm{TeV}$ scale [16]. Indeed, the collision energy can be

\footnotetext{
jys34@yonsei.ac.kr

sc.park@yonsei.ac.kr
}

Published by the American Physical Society under the terms of the Creative Commons Attribution 4.0 International license. Further distribution of this work must maintain attribution to the author(s) and the published article's title, journal citation, and DOI. Funded by SCOAP ${ }^{3}$. as high as $\sqrt{m_{N} E_{\mathrm{CR}, \nu}}=\mathcal{O}(1-100) \mathrm{TeV}$, given that the energy of the colliding cosmic ray or neutrino particle is around $\mathcal{O}(1) \mathrm{PeV}-\mathcal{O}(10) \mathrm{EeV}$ with the nucleon mass $m_{N} \simeq 1 \mathrm{GeV}$.

As a concrete example of relevant physics showing only above $1 \mathrm{TeV}$, we focus on the electroweak sphaleron process in this paper. The sphaleron is predicted to take place at around $10 \mathrm{TeV}$ in the standard model [17-22], but never has been experimentally tested and confirmed yet $[23,24]$. We also study the microscopic black holes predicted in low scale gravity scenarios for the hierarchy problem $[25,26] .{ }^{1}$ The process of sphaleron and that of microscopic black hole share common properties, such as large multiplicities and grown interaction cross sections at higher energies. We show that these common properties lead to observational consequences at the air-shower detector array experiments such as Telescope Array Experiment (TA) and Pierre-Auger Observatory (Auger) [31-40]. Also, black-hole-induced UHE $\nu$-nucleon scattering in the IceCube detector has been studied [41].

The paper is organized as follows: In Sec. II, we discuss the cross sections of electroweak sphaleron and microscopic black hole events. In Sec. III, we discuss the event rates of new physics processes taking the GZK neutrino flux. In Sec. IV, we show noticeable phenomenological features for each case and discuss potential detection of new physics effects. The recently reported "muon deficits" in hadronic interaction models [42-44] are also discussed. Finally, we conclude in Sec. V. The Appendix includes all details of the calculations used in the paper.

\footnotetext{
${ }^{1}$ See [27] for a recent review on microscopic black holes and [28-30] for quantum black holes.
} 


\section{PRODUCTION CROSS SECTIONS FOR SPHALERON AND BLACK HOLE}

In this section, we discuss phenomenological details of the air showers induced by electroweak sphaleron and microscopic black hole. The air showers typically accompany with large multiplicity in signals. The showers are boosted so that the final-state particles are highly collinear and confined within a small separation angle $\delta \theta \sim \mathcal{O}\left(1 / \gamma_{\text {boost }}\right)$, where $\gamma_{\text {boost }}=1 / \sqrt{1-v^{2}}$ is the relativistic gamma factor of the produced particles with velocity $v$.

\section{A. Parton level cross sections}

The electroweak sphaleron is predicted within the standard model as a saddle point solution to the classical field equation of the electroweak gauge theory $[17,18]$. It is a highly unstable configuration thus is not directly observable. However, its decay products, the Standard Model gauge bosons [45], and fermions leave observable effects [46] and reveal their presence in processes in the early universe. It also induces directly measurable signals in high energy collisions of UHE neutrino as we will closely study in this paper.

Sphaleron is involved in baryon number generation by inducing Chern-Simon (CS) number changing $\left(\Delta n_{\mathrm{CS}}= \pm n\right)$ and baryon number $(B)$ and lepton number $(L)$ violating $(\Delta(B+L) \neq 0, \Delta(B-L)=0)$ processes. The generated baryon and lepton numbers are quantized as $\Delta B=\Delta L= \pm 3 n$, where $n \in \mathbb{Z}$ is an integer number. As the sphaleron process is effective in unbroken phase of electroweak symmetry, the baryon number, if generated before electroweak symmetry breaking, will be "wiped out" by sphaleron processes $[19,20]$. On the other hand, the generated lepton number is "converted" into baryon numbers via the sphaleron. Therefore, it is important in baryogenesis from the lepton number generation [20,22].

Beside the role in baryogenesis, even though the sphaleron is robustly predicted within the Standard Model, it has never been experimentally tested in the collider experiments, due to the high sphaleron threshold at $E_{\mathrm{sph}} \approx 10 \mathrm{TeV}$. The UHE neutrinos are particularly interesting since they reach this high threshold. According to recent works by Ellis and his collaborator [47,48], the production cross section of Sphaleron in scattering of $i$ and $j$ initial partons can be relatively large ${ }^{2}$ :

$$
\begin{aligned}
\hat{\sigma}_{i j \rightarrow \operatorname{EWSph}}\left(E_{\mathrm{CM}}\right) & \simeq \frac{p}{m_{W}^{2}} \theta\left(E_{\mathrm{CM}}-E_{\mathrm{sph}}\right), \\
& \equiv \frac{1}{\Lambda_{\mathrm{Sph}}^{2}} \theta\left(E_{\mathrm{CM}}-E_{\mathrm{sph}}\right),
\end{aligned}
$$

\footnotetext{
${ }^{2}$ See, however, [49,50]. Conventionally, it has been believed that the production cross section of a sphaleron in particle collision is exponentially suppressed even when the collision energy is beyond the threshold [22], $\hat{\sigma}_{i j \rightarrow \text { Sphaleron }} \propto e^{-E / E_{\mathrm{sph}}}$.
}

where $m_{W}=80.38 \mathrm{GeV}, \theta(x)$ is the Heaviside step function, and an unknown parameter $p \lesssim 1$ encapsulates the unknown theoretical details. The cross section is not suppressed beyond the threshold because the sphaleron process is a collection of all possible processes over periodic vacua, and the multiple contributions from all vacua overcome the exponential suppression factor [50,51]. We take this cross section as a benchmark expression for the new physics "sphaleron."

A microscopic black hole forms through the collision of particles with a relatively low energy, $\sqrt{s} \gtrsim 1 \mathrm{TeV}$, in lowenergy gravity scenarios $[25,26]$. Once produced, a microscopic black hole may explosively decay into multiple number of photons and also other Standard Model particles through Hawking radiation with high Hawking temperature, $T_{\text {Hawking }}>m_{t}[52,53]$. Thus, the collision of UHE neutrino with a nucleon in the atmosphere is a potential source of a high-multiplicity event.

The parton level production cross section of $(i+j \rightarrow$ black hole $+X)$ is approximately given as

$$
\hat{\sigma}_{\mathrm{ij} \rightarrow \mathrm{BH}}\left(E_{\mathrm{CM}}\right) \approx \pi\left(G_{D} E_{\mathrm{CM}}\right)^{\frac{2}{D-3}},
$$

where $G_{D}=1 / M_{D}^{D-2}$ is the gravitational constant in $D(=4+n)$-dimensions with $n$-extra compact dimensions $[25,26,54,55]$. The Schwarzschild radius is rapidly growing as $r_{\mathrm{Sch}} \approx\left(G_{D} E\right)^{1 / D-3}$ so that the resultant cross section grows too. Assuming $M_{D} \sim \mathrm{TeV}$ in low energy gravity scenarios, the cross section can be sizable as $\hat{\sigma} \gtrsim 1 / \mathrm{TeV}^{2}$ when $E \gtrsim \mathrm{TeV}$.

\section{B. $\nu-N$ cross section}

The $\nu-N$ cross section can be obtained by taking the parton distribution functions (PDF), $f_{q}\left(x, \hat{Q}^{2}\right)$, for a quark, $q$, in nucleon, $N$. The total cross sections for electroweak sphaleron and microscopic black hole are, respectively, given after the PDF convolution for nucleon:

$$
\begin{aligned}
& \sigma_{\text {EWSph,BH }}^{\nu N}\left(E_{\mathrm{lab}}\right) \\
& \quad=\int_{x_{\min }}^{1} d x f_{q}\left(x, 2 x m_{N} E_{\mathrm{lab}}\right) \hat{\sigma}_{\mathrm{EWSph}, \mathrm{BH}}^{\nu q}(\hat{s}),
\end{aligned}
$$

where $\hat{s}=2 x m_{N} E_{\text {lab. }}$. The parton level cross sections are given in Eq. (1) and Eq. (2), respectively. The input parameters are $m_{N}$ (the mass of Nucleon), $E_{\text {lab }}$ (the collision energy in lab frame), and $\hat{s}$ (the collision energy at the parton level). The minimum energy for making a black hole (sphaleron) is controlled by $x_{\min }=\operatorname{Min}\left[\hat{s} /\left(2 m_{N} E_{\text {lab }}\right)\right]$. It is noted that if we set the unknown parameter of the sphaleron cross section $p \sim \mathcal{O}(1)$, the values of cross sections for the sphaleron and black hole are numerically close to each other with $M_{\mathrm{D}} \sim \mathcal{O}(1) \mathrm{TeV}$. Even though the production cross section for black holes eventually overtakes the sphaleron production, the luminosity of UHE 


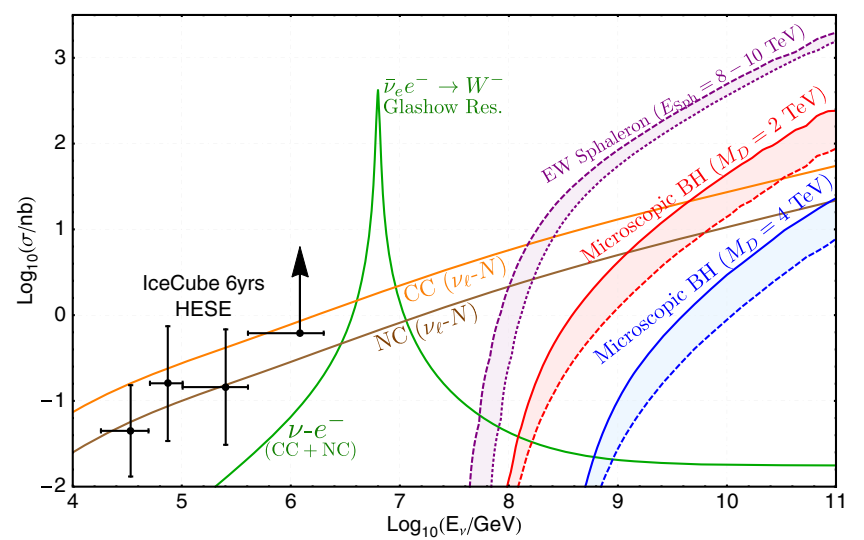

FIG. 1. (Neutrino-induced cross sections with nucleon or electron.) The CC and NC neutrino-nucleon deep-inelastic scattering (DIS) (orange and brown), Glashow resonance (green), electroweak sphaleron with $E_{\mathrm{Sph}}=8-10 \mathrm{TeV}$ (purple band), microscopic black holes with $M_{D}=1 \mathrm{TeV}$ (red band), and $M_{D}=2 \mathrm{TeV}$ (blue band) for the number of compact dimensions, from $n=2$ (bottom) to $n=6$ (top). We fixed the ratio between the minimum energy for black hole production and the gravity scale as $x_{\min } \equiv M_{\min } / M_{D}=5$ for all cases. The black dots with error bars are the $\nu-N$ cross section obtained from the six-year IceCube high-energy starting events (HESE) shower data set.

neutrino becomes smaller at higher energies; therefore, the total event rates stay similar for the two different cases.

In Fig. 1, we show the relative sizes of the standard model NC and CC cross sections [56] and the expected cross sections from sphaleron and black hole with various parameter choices. We use NNPDF 3.1 [57] as the parton distribution function in the evaluation for $\mathrm{CC}$ and $\mathrm{NC}$, sphaleron [48] and the black hole production processes. We find that the sphaleron and black hole interactions will become increasingly important at higher energies as we expected: At low energies below $10^{6-7} \mathrm{GeV}$, the Standard Model CC and NC neutrino-nucleon deep-inelastic scattering (DIS) dominates over other interactions as depicted by orange and brown lines. In a narrow resonance region at the $W$-boson threshold at $E_{\nu}=m_{W}^{2} / 2 m_{e} \simeq 6.3 \mathrm{PeV}$, the $W$ production process, $\bar{\nu}_{e} e^{-} \rightarrow W^{-}$, dominates as depicted by the green, mountain shape line (Glashow resonance). Above $\mathcal{O}(100) \mathrm{PeV}$, the new high multiplicity events, induced by the electroweak sphaleron and black hole, become important and eventually dominate over the standard model CC and NC interactions. ${ }^{3}$ We depict the sphaleron events and black events with the parameters: $E_{\text {Sph }}=8-10 \mathrm{TeV}$ for the sphaleron (purple band) and

\footnotetext{
${ }^{3} \mathrm{CC}$ and NC interaction cross section has dominant contributions at small parton fraction as $x \lesssim 10^{-4}$ and the cross section increases as $\sigma \sim E^{\delta}$, where the parton distribution fitted $x f_{q}(x) \sim x^{-\delta}$. In contrast, new physics (black hole or sphaleron) cross sections come from only large $x$ region as $x \gtrsim x_{\min }=$ $E_{\min }^{2} / 2 m_{N} E_{\nu} \gtrsim 10^{-4}$.
}

$M_{D}=1-2 \mathrm{TeV}$ with fixed $x_{\min } \equiv M_{\min } / M_{D}=5$ for the black hole. We take $M_{D}=1 \mathrm{TeV}, M_{\min }=5 \mathrm{TeV}$ (red band), and $M_{D}=2 \mathrm{TeV}, M_{\min }=10 \mathrm{TeV}$ (blue band) as our benchmark parameter choices. The bands for black hole are for various numbers of extra dimensions $2 \leq n \leq 6$ from bottom $(n=2)$ to top $(n=6)$. Finally, the observational results for the neutrino-nucleon cross section are depicted by black dots with error bars, which are from the six-year IceCube data. In particular, we take the high energy starting events (HESE) [58,59], whose starting points of cascade or track are located inside the IceCube detector.

The proton-proton and proton-air cross sections are large, and the QCD showers are dominant in low $X_{0}$ region. However, we show that the QCD background can be greatly reduced even below the new physics level by imposing a cut in large $X_{0}^{\text {cut }}$ atmospheric depth of injection position. The details are discussed later in Sec. IV C. After the cut, we take the sphaleron-induced and black hole air showers with neutrino-nucleon collision as our signals, and $\mathrm{CC}$ and $\mathrm{NC}$ with neutrino-nucleon collision as our backgrounds.

\section{EVENT RATES AND LIMITS ON HIGH-MULTIPLICITY PROCESSES}

As the fluxes of diffuse gamma rays and the GZK neutrino are correlated, the flux of GZK neutrinos can be determined from the diffuse gamma ray data taken, e.g., by Fermi-LAT [60]. In Fig. 2, we collect the UHE neutrino fluxes obtained from various observational sources in the range of the energy, $E_{\mathrm{sh}} \subset\left(10^{8}, 10^{10}\right) \mathrm{GeV}[10,11]$. In particular, we show the upper bounds on the fluxes obtained from direct measurements from IceCube (20082014) [11] and Pierre Auger (2004-2013) [13]. We also

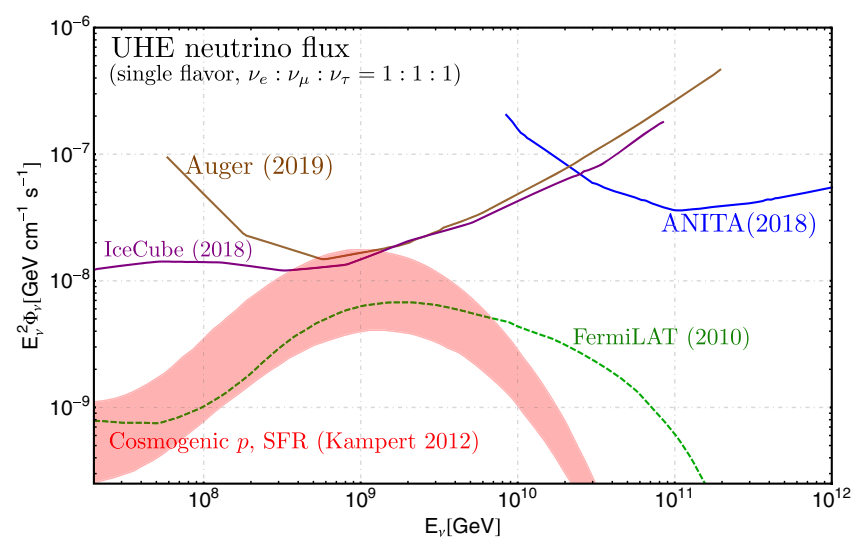

FIG. 2. $E_{\nu}^{2} \Phi_{\nu}$, GZK neutrino fluxes. Expected GZK neutrino fluxes and current experimental bounds. Expected GZK neutrino fluxes from the diffuse gamma-ray observation of Fermi-LAT (green, dashed) [60] and SFR source evolution (red band) [61]. The current experimental bounds from IceCube (purple) [62], ANITA (blue) [63], and Auger (brown) [64]. 

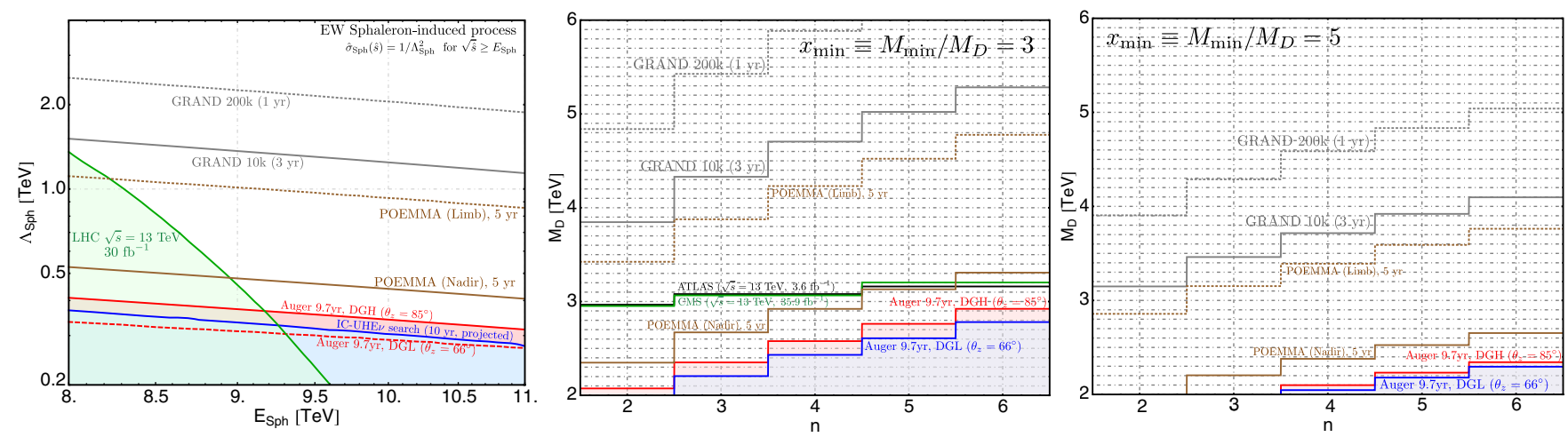

FIG. 3. $90 \%$ confidence level lower limits of the cross section scale for electroweak sphaleron $\Lambda_{\text {Sph }}$ (left panel) and the extradimensional model parameters $\left(n, M_{D}\right)$ for $x_{\min } \equiv M_{\min } / M_{D}=3$ (middle panel), and $x_{\min }=5$ (right panel). For Auger limits, we use the effective area for down-going high zenith $\left(\theta_{z}=85^{\circ}\right.$, DGH) and down-going low zenith $\left(\theta_{z}=66^{\circ}\right.$, DGL) UHE neutrino searches $[65,66]$. We show the current excluded regions by CMS [69], ATLAS [70,71], and IceCube [11]. The expected lower limits in future experiments (GRAND [67] and POEMMA [68]), with several choices of total exposures for UHE $\nu$ search, also are shown.

show the flux obtained from indirect measurement of Fermi-LAT gamma ray data [60] with different minimum energies of extragalactic cosmic rays involved in the photopion production.

With given expectations on UHE neutrino fluxes in Fig. 2, we obtain lower limits of parameters for electroweak sphaleron $\left(\Lambda_{\mathrm{Sph}}\right)$ and extra dimensions $\left(n, M_{D}\right.$ and $\left.M_{\min }\right)$ in Fig. 3. Current observatories such as Auger $[65,66]$ and IceCube [11] and also future proposed facilities (e.g., GRAND [67] and POEMMA [68]) are considered in order to provide limits. For the evaluation of both signal (sphaleron, black hole) and background (electroweak CC and NC) event rates, we follows the expressions in Appendix. A. We use the UHE neutrino flux estimated from Ref. [60] (green dashed line in Fig. 2) as our benchmark choice. For the discrimination between signal and background air showers, we explain the details in Sec. IV B.

On the other hand, CMS [69] and ATLAS [70,71] experiments provide lower limits on the same parameters $\left(\Lambda_{\mathrm{Sph}}, n, M_{D}\right.$ and $\left.M_{\mathrm{min}}\right)$ from the analysis of LHC Run 2 data. However, as shown in Fig. 3, current constraints from LHC are only sensitive for $\sqrt{\hat{s}} \lesssim 10 \mathrm{TeV}$. Typical searches of black hole production only can give exclusion limits for $1<x_{\min } \lesssim 3$, where the semiclassical description of evaporation is not fully guaranteed [72]. In contrast, due to the large (as $\sqrt{s} \simeq 130 \mathrm{TeV}$ for $E_{\nu} \simeq 10^{10} \mathrm{eV}$ ) center-of-mass of expected $\mathrm{UHE} \nu$-nucleon collision in ground array observatories, astrophysical searches can be more relevant for larger $x_{\min }$, up to $x_{\min } \lesssim 5$, as shown in the right panel of Fig. 3.

\section{FEATURES OF NEW PHYSICS EVENTS}

In this section, we describe some evident features of new physics events based on the symmetry principles and the quantum nature of Hawking radiation, which eventually provide useful guidelines to single out the new physics events from the background events.

\section{A. Signals from sphaleron: $(B-L)$ symmetry}

For the sphaleron, due to the symmetries of baryon number and lepton number, $\Delta(B-L)=0$ and $\Delta(B+L)=3 n$ with an integer number $n$, the particle contents of the sphaleron process in the final state are almost uniquely determined. For instance, $\nu_{e}-N$ collision generates (10 fermions $)+\left(n_{B}\right.$ bosons $)$ particles satisfying $\Delta B=$ $\Delta L=-3$. The number of bosons ( $n_{B}$ bosons) is the sum of the number of gauge bosons $\left(n_{W}+n_{Z}\right)$ and the number of Higgs bosons $\left(n_{H}\right)$.

More precisely, if an up-type quark is in the initial nucleon $N$, the final state is

$$
N(u)+\nu_{e} \rightarrow L+Q+n_{W} W+n_{Z} Z+n_{H} H,
$$

where $L(Q)$ stands for the primary leptons (quarks), respectively. For example, $L=\mu^{+}+\bar{\nu}_{\tau}$ and $Q=\bar{t}+2 \bar{b}+$ $2 \bar{c}+\bar{s}+\bar{u}+\bar{d}$ is one of the possible minimal choices satisfying $L=1$ to $L=-2$ and $B=1 / 3$ to $B=-8 / 3$, respectively. In general, there are two antileptons $(L=-2)$ and eight antiquarks $(B=-8 / 3)$ in a final state with possible addition of electroweak bosons. The secondary leptons from the decay of primary (heavy) quarks and gauge bosons are distinguishable as they are less energetic compared to the primary leptons.

Taking all the properties discussed above, we summarize the rules for the signal configuration:

(i) Total $\mathrm{SU}(2)_{L}$ isospin is conserved,

(ii) $\mathrm{SU}(3)$ color is conserved, and the total color is singlet if initial and final states are considered all together,

(iii) $\Delta B_{i}=\Delta L_{j}=\Delta N_{\mathrm{CS}}$ for each families, $(i, j=1$, $2,3)$,

(iv) Total $U(1)_{\mathrm{EM}}$ and $B-L$ charge are conserved. 
Typically, the final state of the sphaleron-induced process consists of $\mathcal{O}(10)$ hadronic jets and a few additional leptons in high energy domain, and each particle carries an energy of about $E \approx M / n_{\text {primary }}$, where $M$ is the new physics scale, and $n_{\text {primary }}$ is the number of primary decay products. The high multiplicity of hadronic components leads to lower individual pion's energy $E_{\pi} \approx$ $E_{\mathrm{CR}} /\left(N_{\pi^{ \pm}}+N_{\pi^{0}}\right)$; thus, the amount of energy loss is relatively smaller before reaching the critical energy, $E_{\text {crit }}=(1-10) \mathrm{GeV}$ [73]. In Fig. 4, we show the number of charged particles (charged particle multiplicity, $N_{\text {Charged }} \simeq N_{\pi^{ \pm}}^{(0)}$ ) from the different origins. The new physics induced air showers have larger $N_{\text {Charged. }}$. We used hadronic interaction models, QGSJET II-04 [74] and EPOS LHC [75] for calculation.

Below the critical energy, the charged pion's decay length, $\gamma c \tau_{\pi^{ \pm}}$, becomes short in comparison to the interaction depth $\lambda_{\pi} \simeq 120 \mathrm{~g} \mathrm{~cm}^{-2}$ of the atmosphere. In this stage, the charged pions decay mainly via $\pi^{+} \rightarrow \mu^{+} \nu_{\mu}$ before they interact with nuclei in air molecules. To some extent, the high multiplicity processes of new physics are similar to the processes due to heavy nuclei in the sense of the superposition model [76]. The extensive air shower from a heavy nuclei of the atomic mass $A_{\mathrm{CR}}$ with primary energy $E_{\mathrm{CR}}$ can be considered as a parallel copy of $A_{\mathrm{CR}}$ proton air showers, and each of the proton carries the primary energy of about $E_{\mathrm{CR}} / A_{\mathrm{CR}}$. It is important to note that the number of muons included in the air shower at the observation level $h_{\mathrm{obs}}$ scales as $N_{\mu} \propto\left(A_{\mathrm{CR}}\right)^{1-\beta}$ [73], where $\beta=\ln _{\frac{3}{2} N_{\pi^{ \pm}}}=0.85$ and $\left(A_{\mathrm{CR}}\right)^{1-\beta} \simeq 1.8$ for the iron nuclei; i.e., $A_{\mathrm{CR}}=56$.

As a summary, the main features of air showers from the different origins are collectively shown in Fig. 5; the schematic picture of each air-shower event from different

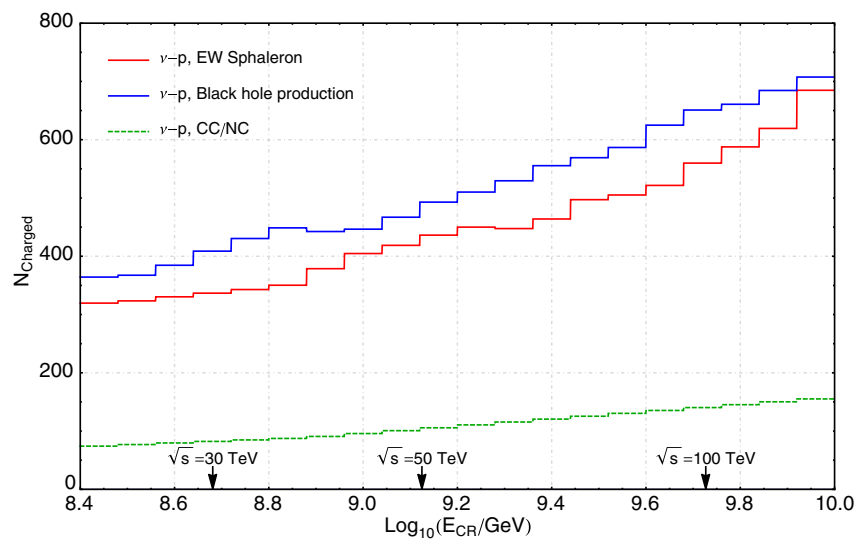

FIG. 4. [Charged particle multiplicity $\left.N_{\text {Charged }}\left(\simeq N_{\pi^{ \pm}}^{(0)}\right)\right]$. The average number of charged particles after the primary hard interactions of neutrino-proton collision with $\mathrm{CC}$ and $\mathrm{NC}$ (green dashed line), EW sphaleron (red solid line) and microscopic black holes productions (blue solid line). primary interactions (sphaleron, black hole, and CC and NC).

\section{B. New Physics air-shower features at ground arrays}

In addition to the high multiplicity of energetic hadronic components $\left(n_{j} \gtrsim 10\right)$ with associated leptons, we also notice distinguishable features of the new physics events by performing realistic simulation of the air-shower events. The simulation is carried out aiming to see the new physics effects in the energy range $10^{15} \mathrm{eV}-10^{20} \mathrm{eV}$ or collision energy of $\sqrt{s}=10-300 \mathrm{TeV}$ ). As a benchmark, we set $E_{\mathrm{Sph}}=9.0 \mathrm{TeV}$ for electroweak sphaleron-induced events. In particular, we consider minimal signals without additional $W$-boson attached. From the events from the microscopic black holes, we set $M_{D}=1-2 \mathrm{TeV}$ and $M_{\min }=5 M_{D}$, which is complimentary to the LHC searches [70,71]. Several MC tools are used for generating the extensive air-shower events: Blackmax [77] for the parton level black hole production and PYTHIA8 [78] for primary parton shower and hadronization. Finally CORSIKA [79] is used for extensive air-shower cascade, where GHEISHA at low energies and QGSJET II-04 [74] (or EPOS LHC [75]) at high energies are attached for hadronic interactions in air-shower cascade simulations. A particularly useful quantity is the atmospheric slant interaction depth, $X(h)$, which is defined as the integrated density of atmosphere along the path of the air shower,

$$
X(h) \equiv \int_{h}^{\infty} d h^{\prime} \rho_{\mathrm{atm}}\left(h^{\prime}\right),
$$

where $\rho_{\text {atm }}(h)$ is the density of the atmosphere at height $h$.

We mainly focus on the observables listed below.

(i) $X_{0}$ (Fig. 7): The first interaction point of air showers, defined as

$$
X_{0} \equiv \int_{h_{\mathrm{inj}}}^{\infty} d h^{\prime} \rho_{\mathrm{atm}}\left(h^{\prime}\right)
$$

where $h_{\mathrm{inj}}$ is the height of the starting point (or injection point) of the air shower induced by the collision of the UHE neutrino and the nucleon in the atmosphere. The typical air-shower events induced by the standard QCD interactions have a relatively steep distribution, but the new physics interactions with a smaller cross section, $\sigma_{\mathrm{NP}} \ll \sigma_{\mathrm{QCD}}$, induce much broader distribution in $X_{0}$ (See Fig. 7), as the probability distribution of the interaction point is given as

$$
P\left(X_{0}\right) \propto \exp \left(-\sigma_{\mathrm{int}} N_{A} A_{\mathrm{atm}}^{-1} X_{0}\right),
$$

where $\sigma_{\text {int }}$ is the cross section of primary interaction, and $A_{\text {atm }}=14$ is the atomic mass of the atmosphere. 


\section{Black hole/Sphaleron}

$\mathrm{CC} / \mathrm{NC}$

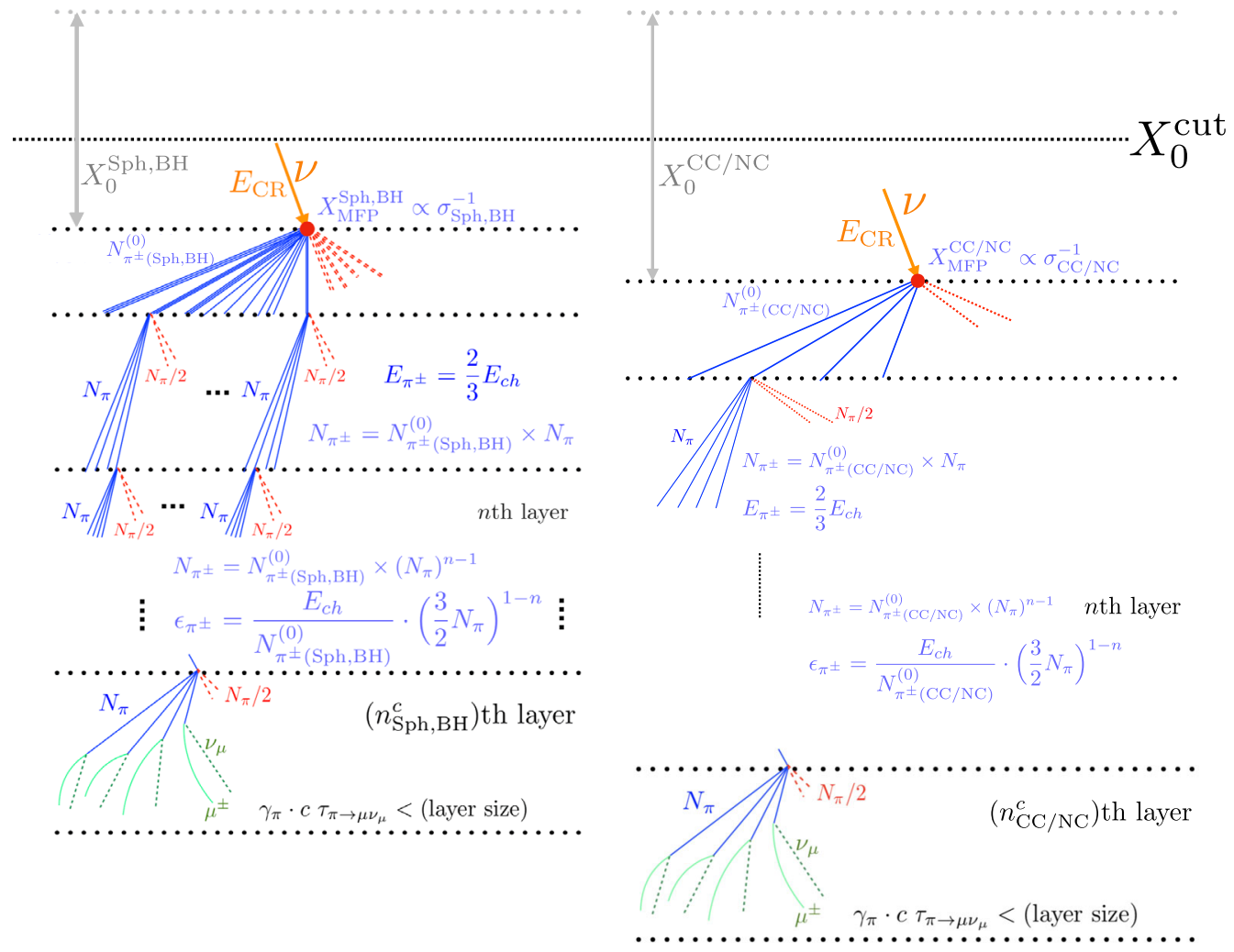

FIG. 5. Schematic view of typical extensive air showers for two cases of primary hard interactions between neutrino and nucleon. Left: EW sphaleron or black hole productions with a primary neutrino and a target nucleon. Right: CC and NC interactions with a primary neutrino and a target nucleon. Note that QCD backgrounds are removed by $X_{0}$ cut. Initial charged pion multiplicity $N_{\pi^{ \pm}(\mathrm{Sph}, \mathrm{BH})}$ and $N_{\pi^{ \pm}(\mathrm{CC} \text { and } \mathrm{NC})}^{(0)}$ are shown in Fig. 4.

(ii) $\Delta X\left(\equiv X_{\max }-X_{0}\right)$ (Fig. 6): $\Delta X$ is the difference between the interaction depth position of the maximum charged particle multiplicity, $X_{\max }$, and the first interaction depth. In this work, we focus on $\Delta X$ because it is highly sensitive to the types of the relevant interactions. $\Delta X$ is observable at 24 fluorescence detector (FD) telescopes in the Auger observatories, for $\theta_{\text {zenith }} \lesssim 60^{\circ}$, by measuring fluorescence light emitted from excited atmospheric (nitrogen) molecules in the range of $300-430 \mathrm{~nm}$ [80]. In addition, Auger can be sensitive to $X_{\max }$ measurement for highly inclined air showers $\left(\theta_{\text {zenith }}=60^{\circ}-84^{\circ}\right)$ by utilizing the radio array facility [81]. Measuring $X_{\max }$ and $\Delta X$ for inclined showers $\left(\theta_{\text {zenith }} \gtrsim 50^{\circ}\right)$ will be crucial to distinguish sphaleron and black hole signal showers from $\mathrm{CC}$ and NC background showers. Nevertheless, detecting the precise value of $\Delta X$ is also important in future searches. GRAND and POEMMA experiments are expected to be sensitive for $X_{\max }$ and $\Delta X$ with the resolution at the level of $\sim 20-40 \mathrm{~g} / \mathrm{cm}^{2}$ $[67,68]$, which is beneficial for giving future limits on sphaleron/black hole showers.
In Fig. 6, we depict the Monte Carlo simulation result of $\Delta X$ for $\mathrm{EW}$ sphaleron (left, red dots with error bars) and black hole (right, blue dots with error bars) in $E_{\mathrm{CR}}=10^{8}-10^{11} \mathrm{GeV}$. All error bars indicate the statistical error of the mean value, which is root-mean-square divided by square-root of the number of entries in each energy bin. Figure 6 indicates $\Delta X$ values for new physics showers, and $\Delta X$ for ordinary $\mathrm{CC}$ and $\mathrm{NC}$ showers are shown in comparison. ${ }^{4}$ We can easily notice that the sphaleron and black hole induced air showers have smaller $\Delta X\left(=X_{\max }-X_{0}\right)$ values compared to $\mathrm{CC}$ and $\mathrm{NC}$ air showers. Our results are consistent with earlier simulation for sphaleron-induced [82] and black-hole-induced [83] air showers. The new physics induced air showers by the sphaleron and black hole have broader distributions in their injection positions $h_{\text {inj. }}$. See Appendix. B for more details).

Imposing $X_{0}^{\text {cut }}$ (Fig. 7) and $\Delta X^{\text {cut }}$ (Fig. 6), our selection criterion for signal is given by

\footnotetext{
${ }^{4} X_{\max }\left(=X_{0}+\Delta X\right)$ distribution for new physics shower will be much broader since $X_{0}$ are almost uniformly distributed.
} 

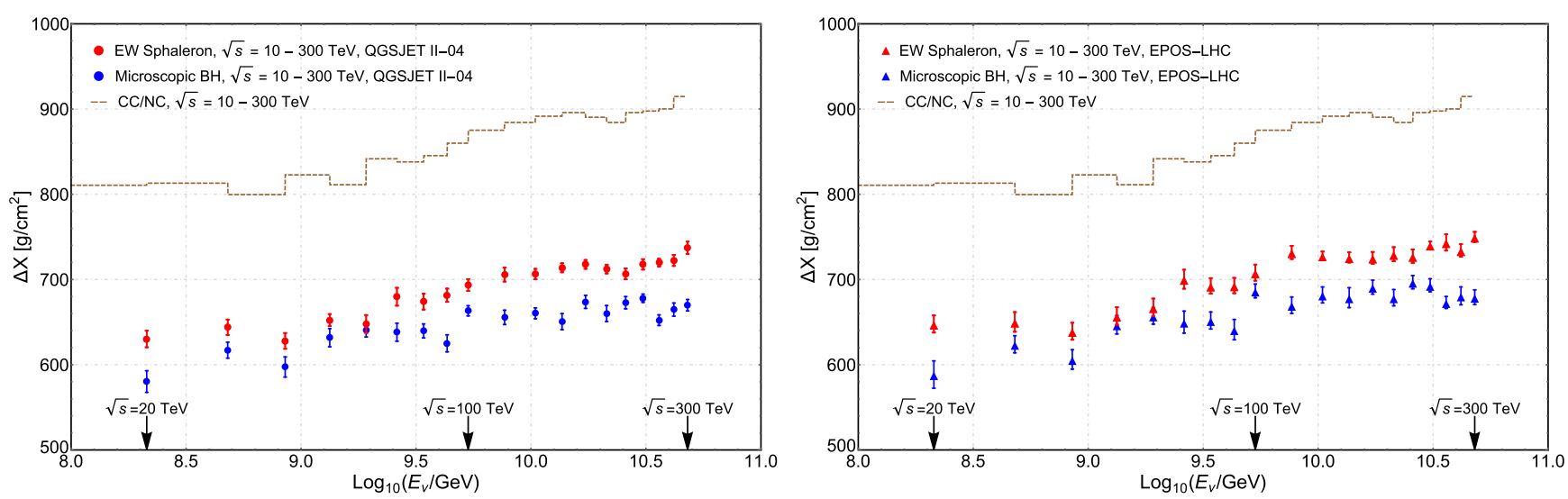

FIG. 6. [The Monte Carlo simulation of expectations of $\Delta X$ for EW sphaleron (red dot), black hole (blue), and CC and NC (brown dashed line) in $E_{\mathrm{CR}}=10^{8}-10^{11} \mathrm{GeV}$.] Note that circles (in the left panel) are the result with QGSJET II-04 [74] and triangles (in the right panel) are with EPOS-LHC [75] in its extensive shower event generation. $\Delta X$ values for CC and NC showers are also shown for comparison.

$$
\text { i) } X_{0}>X_{0}^{\text {cut }}=1200 \mathrm{~g} / \mathrm{cm}^{2} \text {, }
$$

ii) $\Delta X<\Delta X^{\text {cut }} \simeq 50 \log _{10}\left(E_{\mathrm{CR}} / \mathrm{GeV}\right)+260 \mathrm{~g} / \mathrm{cm}^{2}$.

One can expect new physics showers from signal yields under these cuts. Huge charged CR backgrounds can be removed by $X_{0}^{\text {cut }}$ (See Sec. IV C), and new physics showers can be distinguished from remaining $\mathrm{CC}$ and $\mathrm{NC}$ shower backgrounds by considering $\Delta X^{\text {cut }}$. If we consider the view in Fig. 5, we estimate the maximum position as follows [84]:

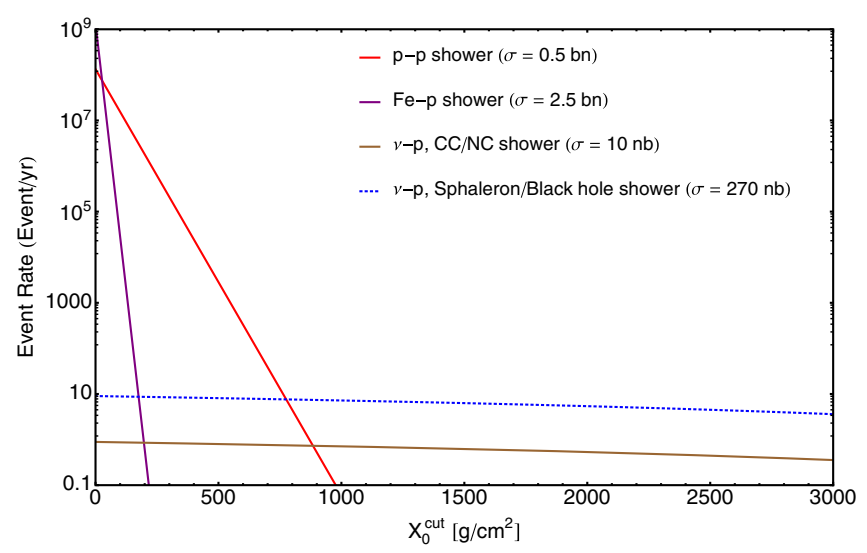

FIG. 7. Event rate on ground detector array after $X_{0}$ cut. Expected number of events per year on Auger after injection point cut $X_{0}^{\text {cut }}$ for primary particle energy $10^{8} \mathrm{GeV}<$ $E_{\mathrm{CR}}<10^{11} \mathrm{GeV}$. New physics event is neutrino-nucleon collision induced by electroweak sphaleron (Eq. (1) with $p=1.0$ ). Total event numbers are obtained for the maximum slant depth $X=5000 \mathrm{~g} / \mathrm{cm}^{2}$, which corresponds to $\theta_{\text {zenith }}=80^{\circ}$. We use (B2) for $X_{0}$ distribution with $\sigma_{\text {int }}$ as each cross sections indicated in the figure.

$$
X_{\mathrm{max}} \simeq X_{0}+X_{\mathrm{MFP}}^{\pi p} \ln \left(\frac{E_{\mathrm{CR}}}{N_{\pi^{ \pm}}^{(0)} \cdot E_{\text {crit. }}}\right),
$$

where $N_{\pi^{ \pm}}^{(0)}$ is the number of charged pions produced in the first hard interactions indicated in Fig. 4 for each interaction, and $X_{\text {MFP }}^{\pi p}=120 \mathrm{~g} / \mathrm{cm}^{2}$ is the mean-free-depth of pions in the atmosphere. $E_{\text {crit }}$ is the minimum energy of charged pions satisfying $\gamma_{\pi} c \tau_{\pi^{ \pm}}<$(layer size), in order to make the pions reinteract with nucleons in air molecules before they decay into muons and neutrinos. As a result, the difference $X_{\max }^{\mathrm{CC}}$ and $\mathrm{NC}-X_{\max }^{\mathrm{NP}}$ is given by

$$
X_{\max }^{\mathrm{CC} \text { and NC }}-X_{\max }^{\mathrm{Sph}, \mathrm{BH}} \simeq X_{\mathrm{MFP}}^{\pi p} \ln \left(\frac{N_{\pi^{ \pm}(\mathrm{Sph}, \mathrm{BH})}^{(0)}}{N_{\pi^{ \pm}(\mathrm{CC} \text { and NC })}^{(0)}}\right) .
$$
Clearly, we notice that the value $\ln \left(\frac{E_{\mathrm{CR}}}{N_{\pi^{ \pm}}^{(0)} \cdot E_{\text {crit. }}}\right)$ corresponds
to the number of layers in Fig. 5.

\section{Cutting the charged CR background events}

In general, the charged CR background events from the collision of UHECR proton and heavy nuclei and nucleon in the atmosphere are dominant sources of shower events observed at a ground based experiment. However, imposing an aggressive cut at a large $X_{0}$ greatly reduces the background as observed in Ref. [33]. Taking the short mean-free-depth $\lambda_{\mathrm{QCD}} \sim 80 \mathrm{~g} / \mathrm{cm}^{2}$ for the QCD event, we found that the cut $X_{0}^{\text {cut }} \gtrsim 1200 \mathrm{~g} / \mathrm{cm}^{2}$ removes almost all QCD backgrounds. The neutrino events, by new physics (black hole, sphaleron) or CC and NC, on the other hand, have longer mean-free depth, and the event distribution is uniform, thus remaining relatively large as clearly seen in Fig. 7. 


\section{The muon number in the UHECR events}

Finally, in this subsection, we address the anomalous result reported by the Pierre Auger collaboration with their nine-year data $[42,43]$ and see the potential account from the new physics effects. The nine-year (2004-2013) Pierre Auger Observatory data set contains many UHE cosmic ray air-shower events, including 29,722 highly inclined events, with the event selection criteria:

(i) $62^{\circ}<\theta_{\text {zenith }}<80^{\circ}$ with $\theta_{\text {zenith }}^{\text {avg. }}=67^{\circ}$,

(ii) $E_{\mu^{ \pm}}>0.3 \mathrm{GeV}$,

(iii) $E_{\mathrm{CR}} \geq 5 \times 10^{18} \mathrm{eV}$.

Among these 29,722 events, there are 174 events indicate the deficit of muons in the Monte Carlo simulations. These highly inclined shower events have more muons than the expected number of muons predicted by the hadronic models. Importantly, the muon number is detectable via SD and FD hybrid detection $[42,43]$. Specifically, $R_{\mu}=$ $N_{\mu} / N_{\mu, 19}$ parameter is used to define the normalized muon number in each extensive air shower event; the total number of muons in each event divided by the reference value of the muon number. We take the reference value $N_{\mu, 19}=2.68 \times$ $10^{7}$ at $\theta_{\text {zenith }}=67^{\circ}$ from Ref. [42], which is obtained from the MC simulation at $E_{\mathrm{CR}}=10^{19} \mathrm{eV}$. We take these events seriously since the similar muon issues are also found in TA 7-years (2008-2015) dataset [44]. The deficit in interaction models mainly appear in highly inclined and highest energies air showers [85]. To account for the muon issue in air-shower events, several approaches have been proposed even though no thorough explanation has been given, including the revision of hadronic interaction models at high energy collisions in the $\sqrt{s}=110-170 \mathrm{TeV}$ range [86] as well as new physics contributions $[87,88]$.

We check if the new physics interactions due to the sphaleron or black hole improves the situation. The results are shown in Fig. 8. The new physics interactions indeed

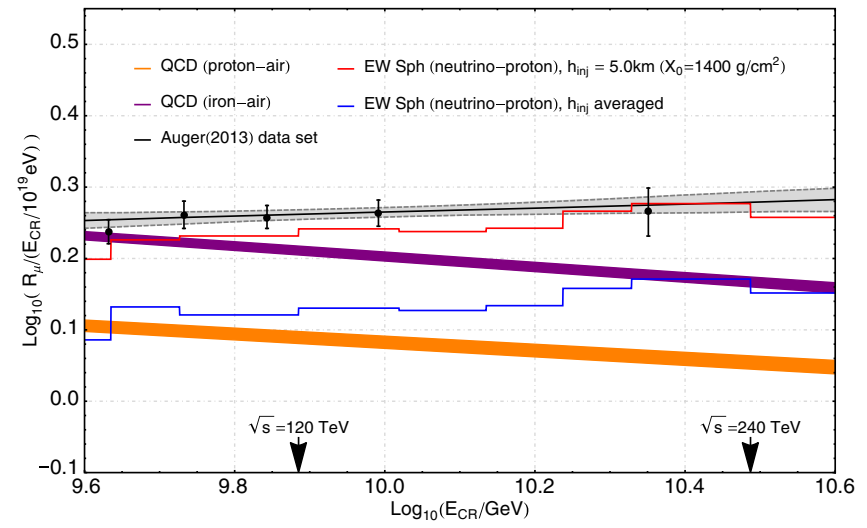

FIG. 8. The total number of muons in highly inclined air showers of nine years of Auger data [42] and the expected muon numbers for sphaleron-induced air showers for a fixed initial injection height $h_{\text {inj }}=5.0 \mathrm{~km}$ of extensive air showers (red solid line). The depth-averaged muon number is also shown (blue solid line). provide some enhancement compared with the QCD events (proton, iron with different modelings) at high energies, $E>10^{9.5} \mathrm{GeV}$. However, when the muon number is averaged over the energy ranges, $R_{\mu}^{\text {avg. }}\left(E_{\mathrm{CR}}\right)$, the new physics interactions do not provide any significant enhancement except for the very deep injection from $h_{\text {inj }} \lesssim$ $5.0 \mathrm{~km}$ (or equivalently, $X_{0} \gtrsim 1400 \mathrm{~g} / \mathrm{cm}^{2}$ ), which is not very likely for the new physics. In conclusion, the new physics interactions by the sphaleron and black hole do not seem to explain the muon deficit in the air showers generated by the hadronic interaction models.

As we mention in Appendix IV C, the muon number measurement for highly inclined air showers still can be informative for new physics search after imposing the $X_{0}$ cut.

\section{CONCLUSION}

Targeting potential new physics located at $\mathcal{O}(10) \mathrm{TeV}$, we studied generic search strategies at the air-shower detector array experiments such as Telescope Array Experiment and Pierre-Auger Observatory, where the collisions of UHE neutrinos and nucleons in the atmosphere are observed. Comparing the signal events with conventional charged $\mathrm{CR}$ and $\mathrm{CC}$ and $\mathrm{NC}$ background events, we found that our target events have larger multiplicities and thus, have characteristic features in showering processes: i) larger $X_{0}$ than charged CR background, ii) larger $N_{\text {Charged }}$ than CC and NC neutrino shower at higher energies, iii) smaller $\Delta X$ than $\mathrm{CC}$ and $\mathrm{NC}$ background, and also distinguishable numbers of electromagnetic, muonic, and hadronic components. The features are highlighted in the schematic figure in Fig. 5. Finally, we also studied potential implication of new physics interactions to the "muon deficit in models" seen in Auger and TA data.

We expect the future air-shower array experiments such as TA $\times 4$ [89], FAST [90], GRAND [91] POEMMA [92], and AugerPrime [93] will reveal the nature of physics at $\mathrm{TeV}$ and beyond.

\section{ACKNOWLEDGMENTS}

This work was supported by the National Research Foundation of Korea (NRF) grants, funded by the Korean government (MSIP) (2021R1A4A2001897) and (2019R1A2C1089334).

\section{APPENDIX A: EVENT RATE IN THE AIR-SHOWER DETECTOR ARRAY}

\section{CC and NC neutrino-induced deep inclined air-shower event}

The CC and NC neutrino-induced, nearly horizontal deep air-shower event rates on the Pierre Auger, for each shower origin, are [56] 
(i) $\mathrm{NC}$ shower for all 3 flavors $\nu_{l=e, \mu, \tau}$ and $\bar{\nu}_{l=e, \mu, \tau}$. $\left(\nu_{l} q \rightarrow \nu_{l} q^{*}\right)$

$$
\begin{aligned}
\frac{d N_{\nu}^{N C}}{d t}= & \rho_{\mathrm{Air}} N_{A} \sum_{l=e, \mu, \tau} \int_{E_{\min }}^{\infty} d E_{\mathrm{sh}} \frac{d \phi_{\nu_{l}}\left(E_{\nu}\right)}{d E_{\nu}} \\
& \times \int_{y_{\min }}^{y_{\max }} d y \frac{d \sigma_{\nu_{l}}^{N C}\left(E_{\nu}, y\right)}{d y} \mathcal{A}\left(E_{\mathrm{sh}}\right)
\end{aligned}
$$

where $E_{\mathrm{sh}}=y E_{\nu}=E_{\nu}-E_{\nu}^{\prime}$ is the total hadronic shower energy.

(ii) $\mathrm{CC}$ shower for $\nu_{e}$ and $\bar{\nu}_{e} \cdot\left(\nu_{e} q \rightarrow e q^{\prime}\right)$,

$$
\begin{aligned}
\frac{d N_{\nu_{e}}^{C C}}{d t}= & \rho_{\mathrm{Air}} N_{A} \int_{E_{\min }}^{\infty} d E_{\mathrm{sh}} \frac{d \phi_{\nu_{e}}\left(E_{\nu}\right)}{d E_{\nu}} \\
& \times \int_{0}^{1} d y \frac{d \sigma_{\nu_{e}}^{C C}\left(E_{\nu}, y\right)}{d y} \Theta\left(E_{\max }-E_{\nu}\right) \mathcal{A}\left(E_{\mathrm{sh}}\right),
\end{aligned}
$$

where $y E_{\nu}=E_{\nu}-E_{e}$, and $E_{e}$ is the hadronic shower and EM shower energy, respectively. The total shower energy is $E_{\mathrm{sh}}=E_{\nu}$.

(iii) $\mathrm{CC}$ shower for $\nu_{\tau}$ and $\bar{\nu}_{\tau}$ with hadronically decaying $\tau\left(\nu_{\tau} q \rightarrow \tau q^{\prime}\right.$, and $\left.\tau \rightarrow \nu_{\tau} q^{\prime \prime} \bar{q}^{\prime \prime \prime}\right)$,

$$
\begin{aligned}
\frac{d N_{\nu_{\tau}}^{C C \text {-had }}}{d t}= & \rho_{\mathrm{Air}} N_{A} \int_{E_{\min }}^{\infty} d E_{\mathrm{sh}} \frac{d \phi_{\nu_{\tau}}\left(E_{\nu}\right)}{d E_{\nu}} \\
& \times \int_{0}^{1} d y \frac{d \sigma_{\nu_{\tau}}^{C C}\left(E_{\nu}, y\right)}{d y} \int_{0}^{1} d z \frac{d n(\tau \rightarrow \mathrm{had})}{d z} \\
& \times \Theta\left(E_{\nu}(y+(1-y)(1-z))-E_{\min }\right) \\
& \times \Theta\left(E_{\max }-E_{\nu}(y+(1-y)(1-z))\right) \\
& \times \operatorname{Br}(\tau \rightarrow \text { hadrons }) \times \mathcal{A}\left(E_{\mathrm{sh}}\right),
\end{aligned}
$$

where $y E_{\nu}=E_{\nu}-E_{\tau}$ is the deposited energy, and $z=E_{\nu}^{\prime} / E_{\tau}$ is the fraction of invisible (neutrino) energy from tau decays, and total shower energy is the sum of hadronic energy of the broken nucleon $y E_{\nu}$ and hadronic energy from tau decays $(1-y)(1-z) E_{\nu}$.

(iv) $\mathrm{CC}$ shower for $\nu_{\tau}$ and $\bar{\nu}_{\tau}$ with electronically decaying $\tau\left(\nu_{\tau} q \rightarrow \tau q^{\prime}\right.$, and $\left.\tau \rightarrow \nu_{\tau} \bar{\nu}_{e} e\right)$,

$$
\begin{aligned}
\frac{d N_{\nu_{\tau}}^{C C-\mathrm{em}}}{d t}= & \rho_{\mathrm{Air}} N_{A} \int_{E_{\min }}^{\infty} d E_{\mathrm{sh}} \frac{d \phi_{\nu_{\tau}}\left(E_{\nu}\right)}{d E_{\nu}} \\
& \times \int_{0}^{1} d y \frac{d \sigma_{\nu_{\tau}}^{C C}\left(E_{\nu}, y\right)}{d y} \int_{0}^{1} d z \frac{d n\left(\tau \rightarrow \nu_{\tau} \bar{\nu}_{e} e\right)}{d z} \\
& \times \Theta\left(E_{\nu}(y+(1-y) z)-E_{\min }\right) \\
& \times \Theta\left(E_{\max }-E_{\nu}(y+(1-y) z)\right) \\
& \times \operatorname{Br}\left(\tau \rightarrow \nu_{\tau} \bar{\nu}_{e} e\right) \times \mathcal{A}\left(E_{\mathrm{sh}}\right),
\end{aligned}
$$

where $y E_{\nu}=E_{\nu}-E_{\tau}$ is the deposited energy again, and $z=E_{e} / E_{\tau}$ is the fraction of EM shower energy from tau decays, and total shower energy is the sum of hadronic energy of broken nucleon $y E_{\nu}$ and tau EM shower energy $(1-y) z E_{\nu}$.

(v) CC shower for $\nu_{\mu}$ and $\bar{\nu}_{\mu} .\left(\nu_{\mu} q \rightarrow \mu q^{\prime}\right)$,

$$
\begin{aligned}
\frac{d N_{\nu_{\mu}}^{C C \text {-had }}}{d t}= & \rho_{\mathrm{Air}} N_{A} \int_{E_{\min }}^{\infty} d E_{\mathrm{sh}} \frac{d \phi_{\nu_{\mu}}\left(E_{\nu}\right)}{d E_{\nu}} \\
& \times \int_{y_{\min }}^{y_{\max }} d y \frac{d \sigma_{\nu_{\mu}}^{C C}\left(E_{\nu}, y\right)}{d y} \mathcal{A}\left(E_{\mathrm{sh}}\right),
\end{aligned}
$$

where $E_{\mathrm{sh}}=y E_{\nu}=E_{\nu}-E_{\mu}$ is the total shower energy.

(vi) CC shower for $\nu_{\tau}$ and $\bar{\nu}_{\tau}$ with muonically decaying $\tau$ $\left(\nu_{\tau} q \rightarrow \tau q^{\prime}\right.$, and $\left.\tau \rightarrow \nu_{\tau} \bar{\nu}_{\mu} \mu\right)$,

$$
\begin{aligned}
\frac{d N_{\nu_{\tau}}^{C C \text {-had }}}{d t}= & \rho_{\operatorname{Air}} N_{A} \int_{E_{\min }}^{\infty} d E_{\mathrm{sh}} \frac{d \phi_{\nu_{\tau}}\left(E_{\nu}\right)}{d E_{\nu}} \\
& \times \operatorname{Br}\left(\tau \rightarrow \nu_{\tau} \bar{\nu}_{\mu} \mu\right) \\
& \times \int_{y_{\min }}^{y_{\max }} d y \frac{d \sigma_{\nu_{\tau}}^{C C}\left(E_{\nu}, y\right)}{d y} \mathcal{A}\left(E_{\mathrm{sh}}\right),
\end{aligned}
$$

where $E_{\mathrm{sh}}=y E_{\nu}=E_{\nu}-E_{\tau}$ is the total shower energy, again.

For the detailed evaluation, we need several quantities defining the detector size, the strength of each interaction and the flux of neutrinos from various sources, such as

(i) $\mathcal{A}\left(E_{\mathrm{sh}}\right)$ is the energy-dependent effective array acceptance, obtained from the effective area $[65,66]$ for UHE neutrino search with $\mathrm{CC} \nu_{e}$ event and rescaled by cross sections for other $\mathrm{CC}$ and $\mathrm{NC}$ and high multiplicity processes. Basically, the Pierre Auger detector array is sensitive above $\mathcal{O}(100) \mathrm{PeV}$, in which GZK neutrinos are dominant.

(ii) $d \sigma_{\nu_{l}}^{C C, N C} / d y$ is the differential CC and NC neutrino $\left(\nu_{l}\right)$-nucleon $(N)$ cross section in the SM [56], respectively, and the total cross sections are

$$
\begin{aligned}
\sigma_{\nu_{l}}^{C C}\left(E_{\nu}\right)= & \frac{2 G_{f}^{2} M_{N} E_{\nu}}{\pi} \int_{0}^{1} d y \int_{0}^{1} d x\left(\frac{M_{W}^{2}}{Q^{2}+M_{W}^{2}}\right)^{2} \\
& \times \sum_{q}\left[x f_{q}\left(x, Q^{2}\right)+x f_{\bar{q}}\left(x, Q^{2}\right)(1-y)^{2}\right], \\
\sigma_{\nu_{l}}^{N C}\left(E_{\nu}\right)= & \frac{G_{F}^{2} M_{N} E_{\nu}}{2 \pi} \int_{0}^{1} d y \int_{0}^{1} d x\left(\frac{M_{Z}^{2}}{Q^{2}+M_{Z}^{2}}\right)^{2} \\
& \times \sum_{q}\left[x f_{q^{0}}\left(x, Q^{2}\right)+x f_{\bar{q}^{0}}\left(x, Q^{2}\right)(1-y)^{2}\right],
\end{aligned}
$$


where $x$ is the parton fraction in the nucleon, and $y$ is the fraction of deposited energy. $f_{q}\left(x, Q^{2}\right)$ and $f_{\bar{q}}\left(x, Q^{2}\right)$ are also defined in [56].

(iii) The energy spectrums $d n / d z$ in electronically [94] and hadronically [95] decaying $\tau$, and the branching ratio in the $\tau$ decay.

(iv) There are neutrinos due to the interaction between high-energy cosmic rays and Earth atmosphere nuclei [96] and the neutrinos of the astrophysical origin, such as highly accelerated hadrons in supernovae remnants, active galactic nuclei (AGN), gamma-ray bursts (GRBs), and shocks in star formation regions of galaxies [97].

(v) Above $\mathcal{O}(100) \mathrm{PeV}$, the UHE neutrinos are produced by the interaction between the UHE cosmic rays (mainly protons and small fractions of other heavy nuclei) above $\mathcal{O}\left(10^{9}\right) \mathrm{GeV}$ and the $\mathrm{CMB}$ photons $[98,99]$, by the following channels: $p+\gamma_{\mathrm{bkg} .} \rightarrow \pi^{+}+n, \pi^{+} \rightarrow \mu^{+}+\nu_{\mu}$ and $p+\gamma_{\mathrm{bkg} .} \rightarrow$ $\pi^{0}+p, \pi^{0} \rightarrow \gamma+\gamma$. This generation of pions is also denoted as the photo-pion production. The cross section for pion production has a resonance peak at the $\Lambda^{+}(1232)$ resonance [100]. These UHE neutrinos from the GZK mechanism are expected from the observations of the UHE cosmic rays by the airshower detector arrays [1,2] and the observations of diffuse photons by the gamma-ray telescopes [60], although there is no direct observation of the GZK neutrinos by the neutrino telescopes yet $[10,11]$.

The UHE neutrinos can produce nearly horizontal and deep air showers, which correspond to $X \sim 13,000 \mathrm{~g} / \mathrm{cm}^{2}$ [12]. For typical flux values and CC and NC interactions, we expect $\sim(0.9-2.9)$ events per year with typical choices of the acceptance values and the GZK neutrino flux models, although no neutrino-induced event candidates have been found yet [101], which provides the bound on the GZK neutrino flux.

\section{Sphaleron and black hole air-shower event}

The event rate in the ground air-shower detector array is given by

$$
\begin{aligned}
\frac{d N}{d t}= & N_{A} \rho_{\mathrm{air}} \int_{E_{\mathrm{th}}}^{E_{\max }} d E_{\mathrm{sh}} \int_{0}^{1} d y \\
& \times \frac{d \phi_{\nu_{l}}\left(E_{\nu}\right)}{d E_{\nu}} \frac{d \sigma_{\nu_{l} X}\left(E_{\nu}, y\right)}{d y} \mathcal{A}\left(E_{\mathrm{sh}}\right),
\end{aligned}
$$

where $\mathcal{A}\left(E_{\mathrm{sh}}\right)$ is the air-shower energy-dependent acceptance of the entire detector array. For Auger, we adopt the values obtained from the effective area $[65,66]$ for $\mathrm{CC} \nu_{e}$ UHE neutrino with rescaling by the sphaleron and black hole cross section.

\section{APPENDIX B: MEAN-FREE-PATH WEIGHTED MUON NUMBER}

The mean-free-path averaged muon number, which is given by

$R_{\mu}^{\text {avg. }}\left(E_{\mathrm{CR}}\right)=\int_{0}^{X_{\text {obs. }}} d X_{0} P\left(X_{0}, \sigma_{\text {int }}\left(E_{\nu}\right)\right) R_{\mu}\left(X_{0}, E_{\nu}\right)$,

where

$$
\begin{aligned}
P\left(X_{0}, \sigma_{\text {int }}\left(E_{\nu}\right)\right)= & \frac{1}{X_{\mathrm{MFP}}\left(\sigma_{\text {int }}\left(E_{\nu}\right)\right)} \\
& \times \exp \left(-X_{0} / X_{\mathrm{MFP}}\left(\sigma_{\text {int }}\left(E_{\nu}\right)\right)\right),
\end{aligned}
$$

and $X_{\mathrm{MFP}}\left(\sigma_{\text {int }}\right)=A_{\mathrm{atm}} \cdot N_{A}^{-1} \cdot \sigma_{\text {int }}^{-1}\left(E_{\nu}\right)$ is the mean-free depth. $A_{\text {atm }}$ is the atomic mass of the atmosphere. Because the cross section for new physics $\sigma_{\text {int }}$ is small enough for our parameter choices, $P\left(X_{0}, \sigma_{\text {int }}\right)$ is almost constant, and the air showers can occur everywhere with almost uniform distribution.
[1] I. Valino (Pierre Auger Collaboration), The flux of ultrahigh energy cosmic rays after ten years of operation of the Pierre Auger Observatory, Proc. Sci., ICRC2015 (2016) 27.

[2] P. Tinyakov (Telescope Array Collaboration), TA anisotropy summary, J. Phys. Soc. Jpn. Conf. Proc. 19, 011019 (2018).

[3] M. G. Aartsen et al. (IceCube Collaboration), First Observation of PeV-Energy Neutrinos with IceCube, Phys. Rev. Lett. 111, 021103 (2013).

[4] P. Blasi, R. I. Epstein, and A. V. Olinto, Ultrahigh-energy cosmic rays from young neutron star winds, Astrophys. J. 533, L123 (2000).
[5] F. Halzen and E. Zas, Neutrino fluxes from active galaxies: A model independent estimate, Astrophys. J. 488, 669 (1997).

[6] E. Waxman, Cosmological origin for cosmic rays above 10**19-eV, Astrophys. J. 452, L1 (1995).

[7] M. Vietri, On the acceleration of ultrahigh-energy cosmic rays in gamma-ray bursts, Astrophys. J. 453, 883 (1995).

[8] D. S. Gorbunov, P. G. Tinyakov, I. I. Tkachev, and S. V. Troitsky, Testing the correlations between ultra-high-energy cosmic rays and BL Lac type objects with HiRes stereoscopic data, JETP Lett. 80, 145 (2004). 
[9] P. Bhattacharjee and G. Sigl, Origin and propagation of extremely high-energy cosmic rays, Phys. Rep. 327, 109 (2000).

[10] M. G. Aartsen et al. (IceCube collaboration), The IceCube neutrino observatory-contributions to ICRC 2015 part II: Atmospheric and astrophysical diffuse neutrino searches of all flavors, in Proceedings, 34th International Cosmic Ray Conference (ICRC 2015): The Hague, The Netherlands, July 30-August 6, 2015, arXiv:1510.05223.

[11] A. Ishihara (IceCube collaboration), Extremely high energy neutrinos in six years of IceCube data, J. Phys. Conf. Ser. 718, 062027 (2016).

[12] P. Abreu et al. (Pierre Auger Collaboration), Ultrahigh energy neutrinos at the Pierre Auger Observatory, Adv. High Energy Phys. 2013, '708680 (2013).

[13] A. Aab et al. (Pierre Auger Collaboration), Improved limit to the diffuse flux of ultrahigh energy neutrinos from the Pierre Auger Observatory, Phys. Rev. D 91, 092008 (2015).

[14] R. U. Abbasi et al. (Telescope Array Collaboration), Depth of ultra high energy cosmic ray induced air shower maxima measured by the telescope array black rock and long ridge FADC fluorescence detectors and surface array in hybrid mode, Astrophys. J. 858, 76 (2018).

[15] Pierre Auger Observatory and Telescope Array: Joint Contributions to the 35th International Cosmic Ray Conference (ICRC 2017) (2018).

[16] L. A. Anchordoqui, Ultra-high-energy cosmic rays, Phys. Rep. 801, 1 (2019).

[17] N. S. Manton, Topology in the Weinberg-Salam Theory, Phys. Rev. D 28, 2019 (1983).

[18] F. R. Klinkhamer and N.S. Manton, A saddle point solution in the Weinberg-Salam theory, Phys. Rev. D 30, 2212 (1984).

[19] V. A. Kuzmin, V. A. Rubakov, and M. E. Shaposhnikov, On the anomalous electroweak Baryon number nonconservation in the early universe, Phys. Lett. 155B, 36 (1985).

[20] M. Fukugita and T. Yanagida, Baryogenesis without grand unification, Phys. Lett. B 174, 45 (1986).

[21] M. E. Shaposhnikov, Baryon asymmetry of the universe in standard electroweak theory, Nucl. Phys. B287, 757 (1987).

[22] A. Ringwald, Sphaleron and level crossing, Phys. Lett. B 213, 61 (1988).

[23] V.A. Rubakov and M. E. Shaposhnikov, Electroweak baryon number nonconservation in the early universe and in high-energy collisions, Usp. Fiz. Nauk 166, 493 (1996).

[24] A. Ringwald, Electroweak instantons/ sphalerons at VLHC?, Phys. Lett. B 555, 227 (2003).

[25] S. Dimopoulos and G. L. Landsberg, Black Holes at the LHC, Phys. Rev. Lett. 87, 161602 (2001).

[26] S. B. Giddings and S. D. Thomas, High-energy colliders as black hole factories: The end of short distance physics, Phys. Rev. D 65, 056010 (2002).

[27] S. C. Park, Black holes and the LHC: A review, Prog. Part. Nucl. Phys. 67, 617 (2012).

[28] N. Arsene, L. I. Caramete, P. B. Denton, and O. Micu, Quantum black holes effects on the shape of extensive air showers, Romanian reports in Physics 69, 105 (2017).
[29] N. Arsene, X. Calmet, L. I. Caramete, and O. Micu, Backto-back black holes decay signature at neutrino observatories, Astropart. Phys. 54, 132 (2014).

[30] N. Arsene, R. Casadio, and O. Micu, Quantum production of black holes at colliders, Eur. Phys. J. C 76, 384 (2016).

[31] T. Ježo, M. Klasen, F. Lyonnet, F. Montanet, I. Schienbein, and M. Tartare, Can new heavy gauge bosons be observed in ultra-high energy cosmic neutrino events?, Phys. Rev. D 89, 077702 (2014).

[32] L. Anchordoqui and H. Goldberg, Experimental signature for black hole production in neutrino air showers, Phys. Rev. D 65, 047502 (2002).

[33] L. A. Anchordoqui, J. L. Feng, H. Goldberg, and A. D. Shapere, Black holes from cosmic rays: Probes of extra dimensions and new limits on TeV scale gravity, Phys. Rev. D 65, 124027 (2002).

[34] R. Emparan, M. Masip, and R. Rattazzi, Cosmic rays as probes of large extra dimensions and $\mathrm{TeV}$ gravity, Phys. Rev. D 65, 064023 (2002).

[35] A. Ringwald and H. Tu, Collider versus cosmic ray sensitivity to black hole production, Phys. Lett. B 525, 135 (2002).

[36] J. L. Feng and A. D. Shapere, Black Hole Production by Cosmic Rays, Phys. Rev. Lett. 88, 021303 (2001).

[37] L. A. Anchordoqui, J. L. Feng, H. Goldberg, and A. D. Shapere, Updated limits on $\mathrm{TeV}$ scale gravity from absence of neutrino cosmic ray showers mediated by black holes, Phys. Rev. D 68, 104025 (2003).

[38] E.-J. Ahn, M. Ave, M. Cavaglia, and A. V. Olinto, TeV black hole fragmentation and detectability in extensive air showers, Phys. Rev. D 68, 043004 (2003).

[39] L. Anchordoqui, T. Han, D. Hooper, and S. Sarkar, Exotic neutrino interactions at the Pierre Auger Observatory, Astropart. Phys. 25, 14 (2006).

[40] K. J. Mack and R. McNees, Bounds on extra dimensions from micro black holes in the context of the metastable Higgs vacuum, Phys. Rev. D 99, 063001 (2019).

[41] K. J. Mack, N. Song, and A. C. Vincent, Signatures of microscopic black holes and extra dimensions at future neutrino telescopes, J. High Energy Phys. 04 (2020) 187.

[42] A. Aab et al. (Pierre Auger Collaboration), Muons in air showers at the Pierre Auger Observatory: Mean number in highly inclined events, Phys. Rev. D 91, 032003 (2015).

[43] A. Aab et al. (Pierre Auger Collaboration), Muons in air showers at the Pierre Auger Observatory: Measurement of atmospheric production depth, Phys. Rev. D 90, 012012 (2014).

[44] R. U. Abbasi et al. (Telescope Array Collaboration), Study of muons from ultrahigh energy cosmic ray air showers measured with the Telescope Array experiment, Phys. Rev. D 98, 022002 (2018).

[45] A. Ringwald, High-energy breakdown of perturbation theory in the electroweak instanton sector, Nucl. Phys. B330, 1 (1990).

[46] D. A. Morris and A. Ringwald, Cosmic ray signatures of multi-W processes, Astropart. Phys. 2, 43 (1994).

[47] J. Ellis and K. Sakurai, Search for sphalerons in protonproton collisions, J. High Energy Phys. 04 (2016) 086. 
[48] J. Ellis, K. Sakurai, and M. Spannowsky, Search for sphalerons: IceCube vs. LHC, J. High Energy Phys. 05 (2016) 085.

[49] K. Funakubo, K. Fuyuto, and E. Senaha, Does a band structure affect sphaleron processes?, arXiv:1612.05431.

[50] S. H. H. Tye and S. S. C. Wong, Baryon number violating scatterings in laboratories, Phys. Rev. D 96, 093004 (2017).

[51] S. H. H. Tye and S. S. C. Wong, Bloch wave function for the periodic sphaleron potential and unsuppressed baryon and lepton number violating processes, Phys. Rev. D 92, 045005 (2015).

[52] S. W. Hawking, Black hole explosions, Nature (London) 248, 30 (1974).

[53] S. W. Hawking, Particle creation by black holes, Commun. Math. Phys. 43, 199 (1975).

[54] S. C. Park and H. S. Song, Production of spinning black holes at colliders, J. Korean Phys. Soc. 43, 30 (2003).

[55] H. Yoshino and Y. Nambu, Black hole formation in the grazing collision of high-energy particles, Phys. Rev. D 67, 024009 (2003).

[56] R. Gandhi, C. Quigg, M.H. Reno, and I. Sarcevic, Neutrino interactions at ultrahigh-energies, Phys. Rev. D 58, 093009 (1998).

[57] R. D. Ball et al. (NNPDF Collaboration), Parton distributions from high-precision collider data, Eur. Phys. J. C 77, 663 (2017).

[58] M. G. Aartsen et al. (IceCube collaboration), Measurement of the multi-TeV neutrino cross section with IceCube using Earth absorption, Nature (London) 551, 596 (2017).

[59] M. Bustamante and A. Connolly, Measurement of the Energy-Dependent Neutrino-Nucleon Cross Section Above $10 \mathrm{TeV}$ Using IceCube Showers, Phys. Rev. Lett. 122, 041101 (2019).

[60] M. Ahlers, L. A. Anchordoqui, M. C. Gonzalez-Garcia, F. Halzen, and S. Sarkar, GZK neutrinos after the Fermi-LAT diffuse photon flux measurement, Astropart. Phys. 34, 106 (2010).

[61] K.-H. Kampert and M. Unger, Measurements of the cosmic ray composition with air shower experiments, Astropart. Phys. 35, 660 (2012).

[62] M. G. Aartsen et al. (IceCube Collaboration), Differential limit on the extremely-high-energy cosmic neutrino flux in the presence of astrophysical background from nine years of IceCube data, Phys. Rev. D 98, 062003 (2018).

[63] P. W. Gorham et al. (ANITA Collaboration), Constraints on the diffuse high-energy neutrino flux from the third flight of ANITA, Phys. Rev. D 98, 022001 (2018).

[64] A. Aab et al. (Pierre Auger Collaboration), Probing the origin of ultra-high-energy cosmic rays with neutrinos in the EeV energy range using the Pierre Auger Observatory, J. Cosmol. Astropart. Phys. 10 (2019) 022.

[65] A. Aab et al. (Pierre Auger collaboration), Limits on pointlike sources of ultra-high-energy neutrinos with the Pierre Auger Observatory, J. Cosmol. Astropart. Phys. 11 (2019) 004.

[66] The Pierre Auger Observatory: Contributions to the 36th International Cosmic Ray Conference (ICRC 2019): Madison, Wisconsin, USA, July 24- August 1, 2019 (2019).
[67] J. Álvarez-Muñiz et al. (GRAND Collaboration), The giant radio array for neutrino detection (GRAND): Science and design, Sci. China Phys. Mech. Astron. 63, 219501 (2020).

[68] A. V. Olinto et al., The POEMMA (Probe of Extreme Multi-Messenger Astrophysics) observatory, J. Cosmol. Astropart. Phys. 06 (2021) 007.

[69] A. M. Sirunyan et al. (CMS Collaboration), Search for black holes and sphalerons in high-multiplicity final states in proton-proton collisions at $\sqrt{s}=13 \mathrm{TeV}$, J. High Energy Phys. 11 (2018) 042.

[70] G. Aad et al. (ATLAS Collaboration), Search for strong gravity in multijet final states produced in pp collisions at $\sqrt{s}=13 \mathrm{TeV}$ using the ATLAS detector at the LHC, J. High Energy Phys. 03 (2016) 026.

[71] M. Aaboud et al. (ATLAS Collaboration), Search for TeVscale gravity signatures in high-mass final states with leptons and jets with the ATLAS detector at $\sqrt{s}=13 \mathrm{TeV}$, Phys. Lett. B 760, 520 (2016).

[72] S. C. Park, Critical comment on the recent microscopic black hole search at the LHC, Phys. Lett. B 701, 587 (2011).

[73] J. Matthews, A Heitler model of extensive air showers, Astropart. Phys. 22, 387 (2005).

[74] S. Ostapchenko, Monte Carlo treatment of hadronic interactions in enhanced Pomeron scheme: I. QGSJET-II model, Phys. Rev. D 83, 014018 (2011).

[75] T. Pierog, I. Karpenko, J. M. Katzy, E. Yatsenko, and K. Werner, EPOS LHC: Test of collective hadronization with data measured at the CERN Large Hadron Collider, Phys. Rev. C 92, 034906 (2015).

[76] J. Engel, T. K. Gaisser, T. Stanev, and P. Lipari, Nucleusnucleus collisions and interpretation of cosmic ray cascades, Phys. Rev. D 46, 5013 (1992).

[77] D.-C. Dai, G. Starkman, D. Stojkovic, C. Issever, E. Rizvi, and J. Tseng, BlackMax: A black-hole event generator with rotation, recoil, split branes, and brane tension, Phys. Rev. D 77, 076007 (2008).

[78] T. Sjostrand, S. Mrenna, and P.Z. Skands, A brief introduction to PYTHIA8.1, Comput. Phys. Commun. 178, 852 (2008).

[79] D. Heck, G. Schatz, J. Knapp, T. Thouw, and J. Capdevielle, CORSIKA: A Monte Carlo code to simulate extensive air showers.

[80] J. Abraham et al. (Pierre Auger Collaboration), The fluorescence detector of the Pierre Auger Observatory, Nucl. Instrum. Methods Phys. Res., Sect. A 620, 227 (2010).

[81] A. Aab et al. (Pierre Auger Collaboration), Observation of inclined $\mathrm{EeV}$ air showers with the radio detector of the Pierre Auger Observatory, J. Cosmol. Astropart. Phys. 10 (2018) 026.

[82] G. Brooijmans, P. Schichtel, and M. Spannowsky, Cosmic ray air showers from sphalerons, Phys. Lett. B 761, 213 (2016).

[83] E.-J. Ahn and M. Cavaglia, Simulations of black hole air showers in cosmic ray detectors, Phys. Rev. D 73, 042002 (2006).

[84] S. Mollerach and E. Roulet, Progress in high-energy cosmic ray physics, Prog. Part. Nucl. Phys. 98, 85 (2018).

[85] Yu. A. Fomin, N. N. Kalmykov, I. S. Karpikov, G. V. Kulikov, M. Yu. Kuznetsov, G. I. Rubtsov, V. P. Sulakov, 
and S. V. Troitsky, No muon excess in extensive air showers at 100-500 PeV primary energy: EAS-MSU results, Astropart. Phys. 92, 1 (2017).

[86] A. Aab et al. (Pierre Auger Collaboration), Testing Hadronic Interactions at Ultrahigh Energies with Air Showers Measured by the Pierre Auger Observatory, Phys. Rev. Lett. 117, 192001 (2016).

[87] G. R. Farrar and J. D. Allen, A new physical phenomenon in ultra-high energy collisions, EPJ Web Conf. 53, 07007 (2013).

[88] J. Alvarez-Muniz, L. Cazon, R. Conceicao, J. D. de Deus, C. Pajares, and M. Pimenta, Muon production and string percolation effects in cosmic rays at the highest energies, arXiv:1209.6474.

[89] H. Sagawa (Telescope Array Collaboration), Telescope array extension, Nucl. Part. Phys. Proc. 279-281, 145 (2016).

[90] T. Fujii et al., Detection of ultra-high energy cosmic ray showers with a single-pixel fluorescence telescope, Astropart. Phys. 74, 64 (2016).

[91] K. Fang et al., The giant radio array for neutrino detection (GRAND): Present and perspectives, Proc. Sci., ICRC2017 (2017) 996.

[92] A. V. Olinto et al., POEMMA: Probe of extreme multimessenger astrophysics, Proc. Sci., ICRC2017 (2017) 542.
[93] A. Aab et al. (Pierre Auger Collaboration), The Pierre Auger Observatory upgrade_-preliminary design report, arXiv:1604.03637.

[94] F. Scheck, Muon physics, Phys. Rep. 44, 187 (1978).

[95] M. Davier, L. Duflot, F. Le Diberder, and A. Rouge, The optimal method for the measurement of tau polarization, Phys. Lett. B 306, 411 (1993).

[96] M. G. Aartsen et al. (IceCube Collaboration), Search for neutrino-induced particle showers with IceCube-40, Phys. Rev. D 89, 102001 (2014).

[97] M. G. Aartsen et al. (IceCube Collaboration), A combined maximum-likelihood analysis of the high-energy astrophysical neutrino flux measured with IceCube, Astrophys. J. 809, 98 (2015).

[98] K. Greisen, End to the Cosmic Ray Spectrum?, Phys. Rev. Lett. 16, 748 (1966).

[99] G. T. Zatsepin and V.A. Kuzmin, Upper limit of the spectrum of cosmic rays, JETP Lett. 4, 78 (1966).

[100] K. K. Andersen and S. R. Klein, High energy cosmic-ray interactions with particles from the Sun, Phys. Rev. D 83, 103519 (2011).

[101] G. Parente and E. Zas, Neutrino induced events in the Pierre Auger detector, in 7th International Workshop on Neutrino Telescopes Venice, Italy, February 27-March 1, 1996 (1996). 